\title{
Deuterium Plasma Focus Measurements using Solid State Nuclear Track Detectors
}

\author{
S.V. Springham, S. Lee, and S.P. Moo* \\ Natural Sciences, National Institute of Education, \\ Nanyang Technological University, 1 Nanyang Walk, Singapore 637616
}

Received on 26 June, 2001

\begin{abstract}
A camera obscura technique was used to investigate the spatial distribution of fusion protons emitted from a small deuterium plasma focus device operated in its neutron-optimized regime $(14 \mathrm{kV}, 3 \mathrm{~kJ}$, and $400 \mathrm{~Pa}_{2}$ gas). An indium-foil activation detector was employed simultaneously to measure the shot-to-shot neutron yield. The camera obscura was positioned on the forward axis of the plasma focus, $12 \mathrm{~cm}$ from the tip of the hollow copper anode, covering a conical field-of-view of half-angle slightly less than 20 degrees. The nuclear track detector material PM-355 was used in the camera focal plane to register protons from the ${ }^{2} \mathrm{H}(d, p)^{3} \mathrm{H}$ reaction, hence imaging the spatial distribution of fusion occurring in the plasma focus. A kapton film covering the camera entrance pupil served to protect the detector from the hot plasma jet; it also stopped completely all energetic charged particles other than the $(\simeq 3 \mathrm{MeV})$ fusion protons. Following chemical etching of the detector plates, the track parameters - size, circularity, average-grey-value and $(x-y)$ position - were measured by an automated scanning system comprising an optical microscope equiped with a CCD camera, motorized stage and focus-adjust, and interfaced to a PC computer. From the accumulated track data, representing 119 plasma focus shots, the imaged fusion density profile was obtained. A detailed Monte Carlo simulation program was used to interpret this profile. The results indicate that the beam-target mechanism, in which energetic deuterons emitted in a forwardly directed cone from the plasma focus pinch interact with the cold deuterium gas, accounts for 90 to $96 \%$ of the fusion production for our device. There is however clear evidence for a non-zero (4 to $10 \%$ ) contribution emitted directly from the pinch region.
\end{abstract}

\section{Introduction}

The plasma focus (PF) was discovered almost four decades ago[1,2] and immediately attracted the interest of researchers due to the intense bursts of neutrons emitted-from the ${ }^{2} \mathrm{H}(d, n){ }^{3} \mathrm{He}$ fusion reaction-when the device is operated in deuterium gas. A large number of experimental investigations have since been conducted with the aim of better understanding the nature of the fusion mechanism, and especially to test the validity of the thermonuclear model and various competing models based on supra-thermal mechanisms, e.g. the beam-target[3, 4] and gyrating-particle[5] models. These studies have concentrated mainly on neutron fluence characteristics, including neutron yield and anisotropy, neutron energy, temporal pulse duration and shape $[3,6,7]$. In particular, the neutron pulse duration $(\sim 100 \mathrm{~ns})$ and the observed neutron anisotropy provide compelling evidence that supra-thermal mechanisms play an important role, yet a detailed understanding of PF fusion is still lacking.

In contrast to the neutron measurements, a paucity of experimental data exists on the charged fusion products-protons, tritons, and ${ }^{3} \mathrm{He}$-although as pointed out by Jäger et al. [8], such studies could provide much needed additional information on the fusion mechanism. In the present work the spatial distribution of protons from the ${ }^{2} \mathrm{H}(d, p)^{3} \mathrm{H}$ reaction is studied using a camera obscura technique. The fusion protons, with energies of approximately $3 \mathrm{MeV}$, are far more penetrating than any of the other charged particles and are therefore more easily distinguished and studied. Of course, a detector for charged particles must be placed inside the PF chamber, which represents a very hostile environment for almost all common charged particle detectors (e.g. silicon surface barrier detectors). The adverse environmental conditions encountered during a PF shot include: intense emissions of light, ultra-violet, $\mathrm{x}$-rays and energetic electrons; a strong axially directed deuteron beam followed by a hot plasma jet; and strong electromagnetic noise. Moreover, the charged particle emission occurs on a time scale of less than $10^{-7} \mathrm{~s}$, hence the count-rate requirement for an electronic de-

\footnotetext{
* On leave from University of Malaya, Kuala Lumpur, Malaysia.
} 
tector would be extremely high.

In view of these considerations, it is apparent that polymer solid-state nuclear track detectors are well suited to charged particle studies of plasma focus fusion. Charged particles incident on a polymer track detector, such as CR-39/PM-355, deposit their kinetic energy as dense trails of ionisation and excitation resulting in numerous polymer chain scissions. These damage trails represent latent particle tracks which can be made visible by chemical etching at some time (perhaps hours or years) after the exposure[9]. The material used throughout this work is PM-355 (Pershore Mouldings, UK), which is a super-grade form of CR-39. The principal advantage of PM-355 is that it is very sensitive to low- $Z$ ions over a wide range of energies[10], yet is insensitive to light, UV, x-rays, etc. For the fusion protons encountered in these experiments PM-355 has a detection efficiency of $\simeq 100 \%$, and the spatial resolution associated with track position is superb, being approximately 2 microns. Probably the main impediment to using track detectors is the difficulty of reading the track information from the detector plates. Counting etched tracks by eye using an optical microscope has been the most frequently employed method for many years. Naturally, the tedious and laborious nature of such work is not consistent with high accuracy or the analysis of a large number of detectors. And if the spatial distribution of tracks is required, rather than simply the gross count, the task of manual analysis becomes very onerous. In the present work, an automated track measurement system has been employed which overcomes the limitations of manual counting.

\section{The Experimental Setup}

The plasma focus used in this work is a $3 \mathrm{~kJ}$ UNU/ICTP PFF $[11,12]$ of Mather-type geometry. The hollow copper anode has a length $160 \mathrm{~mm}$ and diameter $19 \mathrm{~mm}$. The co-axial cathode comprises six copper rods arranged in a squirrel cage configuration at a radius of $3.2 \mathrm{~cm}$. An annular pyrex tube located at the base of the anode insulates it from the cathode and plays an important role in ensuring the symmetry of the initial gas breakdown. Energy storage is provided by a $32 \mu \mathrm{m}$ capacitor and the discharge is triggered by a simple parallel-plate spark gap with a swinging-cascade configuration. Throughout the present work the plasma focus was operated in its neutron optimised regime, corresponding to $400 \mathrm{~Pa}$ deuterium gas pressure and a charging voltage of $14 \mathrm{kV}$. In this regime a peak focus current of $180 \mathrm{kA}$ and an average shot-to-shot neutron yield of $\sim 10^{8}$ are obtained. The electrical diagnostics employed were a Rogowski coil for the focus current, and a voltage probe comprising a high-voltage resistive divider. An indium foil activation counter was used to measure the neutron yield. The relevant dimensions of the plasma focus chamber are: diameter $160 \mathrm{~mm}$ and anode to top-plate distance $170 \mathrm{~mm}$.

The proton camera obscura was positioned on the forward axis of the plasma focus with its front face at distance of $120 \mathrm{~mm}$ from the tip of the anode. The $1.8 \mathrm{~mm}$ diameter entrance pupil (through $75 \mu \mathrm{m}$ thick brass) was filtered by a $50 \mu \mathrm{m}$ kapton film. PM-355 detector plates were placed in contact with the camera exit pupil (entrance to exit pupil distance $34 \mathrm{~mm}$, diameter $20.4 \mathrm{~mm}$ ) resulting in an approximate angular field of view of $20^{\circ}$ with respect to the camera axis. The performance of the camera was checked using the TRIM "Trajectories of Ions in Matter" simulation code[13]. For a deuteron energy of $50 \mathrm{keV}$ the protons emitted in the forward direction from the ${ }^{2} \mathrm{H}(d, p){ }^{3} \mathrm{H}$ reaction have an energy of $3.3 \mathrm{MeV}$; these protons will have an energy of about $2.4 \mathrm{MeV}$ after passing through the $50 \mu \mathrm{m}$ thick kapton window; their residual range in brass being approximately $26 \mu \mathrm{m}$. The other charged particles resulting from fusion reactions: ${ }^{3} \mathrm{He}(\sim 0.8 \mathrm{MeV})$ and ${ }^{3} \mathrm{H}(\sim 1 \mathrm{MeV})$ will easily be stopped in the kapton film, along with the large flux of low energy deuterons. To penetrate the kapton window, deuterons would require energies greater than $2.5 \mathrm{MeV}$ and the experimental results provide no evidence that such energetic deuterons are emitted from our PF device. In passing through the kapton window the fusion protons suffer an average angular deviation of only $1.2^{\circ}$, hence this arrangement acts very effectively as a camera obscura which images the fusion protons on the PM-355 detector plate.

The automated track measurement system[14] we have developed comprises a Leica DMRB optical microscope with CCD camera, motorised microscope stage ( $x-y$ axes), motorised focussing ( $z$ axis), and motorcontroller unit. The motor-controller unit and CCD camera are interfaced to a PC running a commercial image processing software (Optimas 6.1).

\section{Experimental Procedure and Track Detector Analysis}

It was found that a satisfactory density of tracks on the detector was obtained for 5 to $7 \mathrm{PF}$ shots; the actual number of shots fired for each detector being judged by the accumulated count from the indium activation detector. A total of 18 detectors were exposed in this manner, representing an accumulated $119 \mathrm{PF}$ shots. The PM-355 detectors were etched in 6.25 molar $\mathrm{NaOH}$ solution at $90^{\circ} \mathrm{C}$ for five hours. The etched proton tracks had diameters in the range 16 to 18 microns. Visual examination of the detectors under the microscope revealed that: in the central region of the expo- 
sure (corresponding to near normally incident protons) the tracks appeared perfectly circular and uniformly dark; towards the edge of the exposure (for which the proton angle of incidence approaches $20^{\circ}$ ) the tracks appeared slightly elliptical and less dark.

The detectors were scanned at $\times 100$ magnification, for which the CCD field of view is a rectangle with dimensions $982 \mu \mathrm{m} \times 738 \mu \mathrm{m}$. The scan path was a $22 \times 28$ raster pattern, resulting in a nearly square scan area into which the circular exposure on the PM355 detector could reliably fit. For each CCD image of the detector surface, the image analysis software applied a local threshold technique to recognise dark features (representing candidate proton tracks), and defined a feature-boundary for each. The scanning system then calculated and recorded for each feature: i) the absolute $(x-y)$ centroid position in microns, ii) the average-grey-value $(\mathrm{AGV}=0-255)$ of the enclosed pixels, iii) the area-equivalent-diameter (AED $=2 \times \sqrt{\text { Area } / \pi})$ in pixels, and iv) the circularity parameter $\left(\mathrm{CP}=(\text { Perimeter })^{2} /\right.$ Area $)$.

Figs. 1 and 2 show data for the above set of parameters, acquired from one PM-355 detector exposed to 7 plasma focus shots. Fig. 1(b) shows a "raw data" scatter plot of the positions of dark features (i.e. candidate tracks) as recognised by the scanning system. For the recognition of candidate track features, the scanning system applied criteria which were intentionally chosen to be fairly un-discerning. This was done in order to minimise the number of genuine proton tracks excluded from the data during scanning. In the subsequent analysis, stricter criteria were imposed on the extracted parameters $\{\mathrm{AGV}, \mathrm{AED}, \mathrm{CP}\}$ on a feature- by-feature basis, in order to filter out spurious tracks and as far as possible accept only genuine proton tracks. Fig. 1(a) shows a scatter plot of this filtered data, and it is evident that the great majority of features lying outside the exposed circular area have been removed from the data. Due to variations in detector etching, the criteria imposed for the selection of genuine proton tracks are slightly different for each detector. For the case shown in Figs. 1 and 2, the applied criteria were: $\{\mathrm{AGV}<120,13<\mathrm{AED}<19, \mathrm{CP} \leq 14\}$. For example, of the 6352 dark features initially measured by the scanning system for this detector, 4135 met the criteria for acceptance as proton tracks, and 4010 of these lie within the exposed area. The remaining features outside the exposed area are due to: a) proton-recoil tracks resulting from the scattering of $\sim 2.5 \mathrm{MeV}$ fusion neutrons within the detector material, and b) spurious track-like flaws and scratches on the detector surface; these represent a "false-track" background distributed uniformly over the detector surface. In order to check the level of this background, several detectors were half covered with a $200 \mu \mathrm{m}$ thick film of PTFE before being exposed, etched, and analysed by the same procedure as the uncovered detectors. PTFE is a hydrogen-free polymer and so does not act as a radiator of recoil-protons; it is also relatively dense, and a $200 \mu \mathrm{m}$ layer is sufficient to stop all the fusion protons. Fig. 3 shows the resulting track distribution for one PTFE half-covered detector. The areal density of these false-tracks is found to be sufficiently low that it can be neglected in the subsequent analysis.
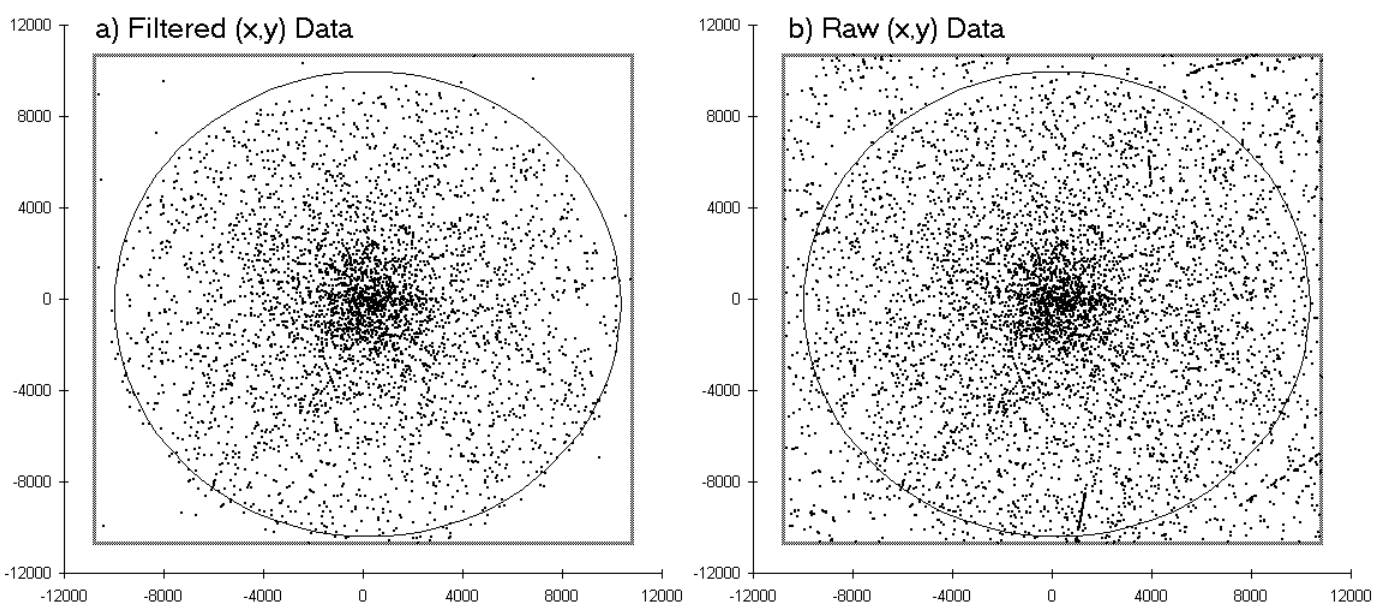

Figure 1. Scatter plots of the spatial distribution of dark features / proton tracks on the surface of one PM-355 detector. Distances shown on the axes are in microns. The (almost square) rectangle represents the area scanned by the automated system, and the circle represents the exposed area of the PM-355 detector (conterminous with the camera exit pupil). (a) Distribution of accepted proton tracks. (b) Distribution of all candidate features on the detector surface. 


\section{Data Analysis, Results and Discussion}

A Monte Carlo simulation program was written to predict the radial distribution of tracks in the detector plane for a beam of deuterons, of initial energy $E_{\mathrm{d}}$, emitted isotropically within a cone of half-angle $\phi_{0}$. Deuterons emerging from the pinch region travel in essentially straight-line trajectories, though gradually slowing down, and producing a conical volume of fusion via the beam-target interaction with the cold deuterium gas. The deviation of deuteron paths from straight-line trajectories, due to multiple small-angle scattering, was checked using the TRIM simulation code and found to be negligible. For example, $50 \mathrm{keV}$ deuterons traversing a $10 \mathrm{~cm}$ path of $\mathrm{D}_{2}$ gas at $400 \mathrm{~Pa}$ suffer an average deviation of only $0.59 \mathrm{~mm}$. They also lose $\approx 9.7 \mathrm{keV}$ of their kinetic energy, with consequent reductions in the $(d, n)$ and $(d, p)$ cross-sections of $41 \%$ and $40 \%$, respectively [Table II, Ref. 15.]
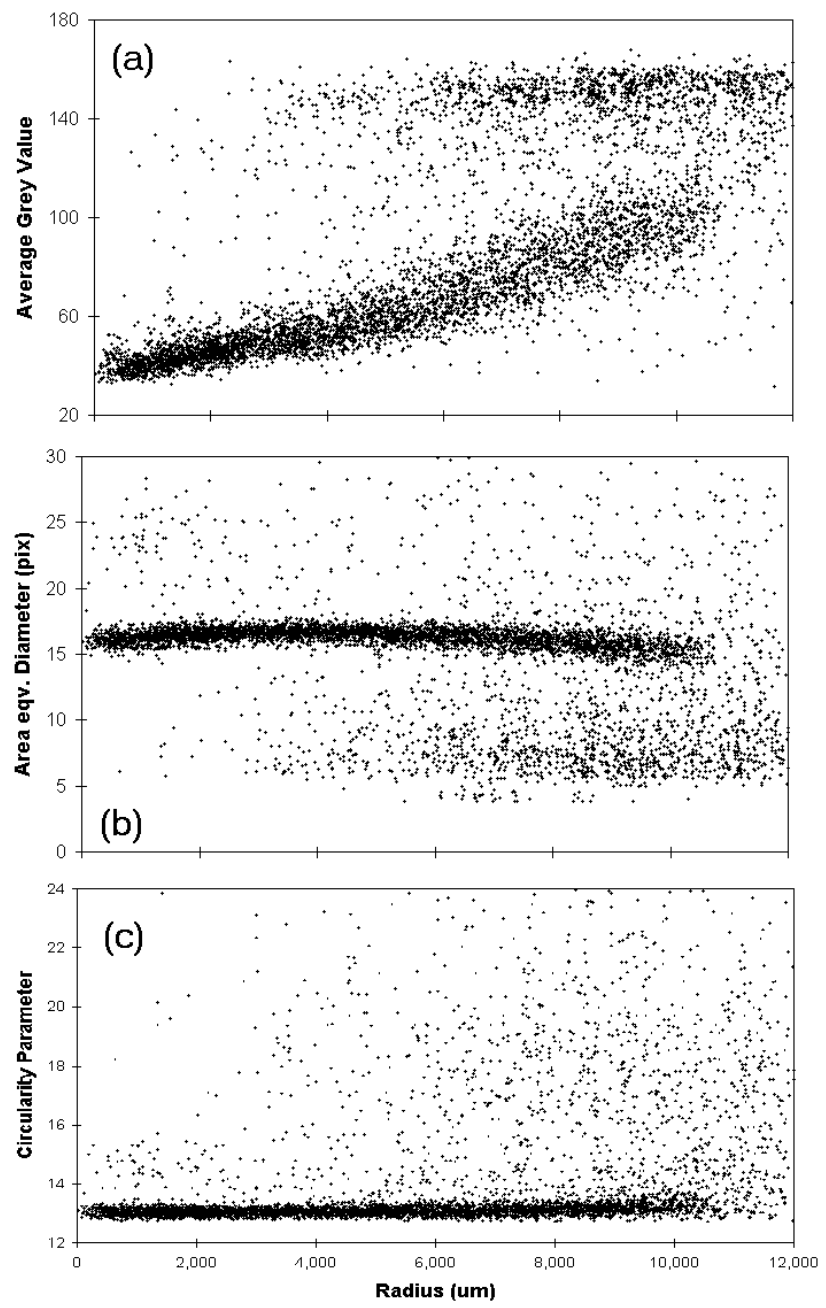

Figure 2. Scatter plots of extracted feature parameters versus radius: (a) average grey value: $\mathrm{AGV},(\mathrm{b})$ area equivalent diameter: AED, and (c) circularity parameter: CP.

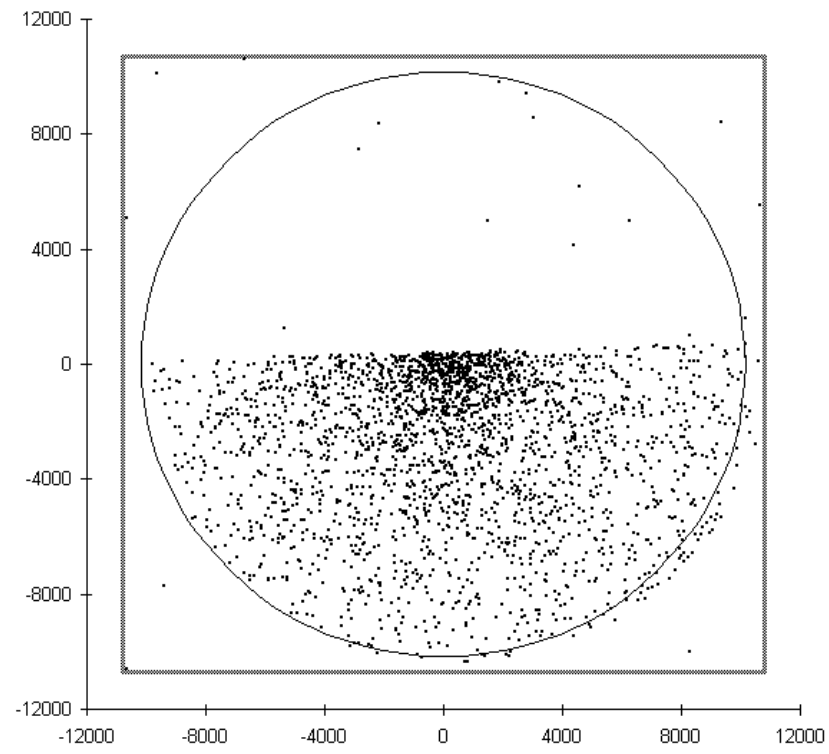

Figure 3. Scatter plot of recognised proton tracks on a PM355 detector with its surface half covered by a 200 micron thick film of PTFE during exposure.

Our Monte Carlo simulation program makes use of the ${ }^{2} \mathrm{H}(d, p)^{3} \mathrm{H}$ differential cross-section and ${ }^{2} \mathrm{H}(d, n)^{3} \mathrm{He}$ total cross-section parameterisations given in Ref. 15. The deuteron energy-loss values are calculated from TRIM, and the appropriate centre-of-mass to lab-frame transformation is applied to the ${ }^{2} \mathrm{H}(d, p)^{3} \mathrm{H}$ differential cross-section. Our simulation takes full account the geometry of the PF chamber and the camera obscura, including small angle scattering of protons in the kapton window. The program inputs are: initial deuteron energy $E_{\mathrm{d}}$, cone half-angle $\phi_{0}$, and the neutron yield $Y_{\mathrm{n}}$. The simulation calculates: (1) the number of beam deuterons; (2) the radial track density histogram for a beam-target (BT) fusion source; and (3) a similar histogram for an isotropic point-source (PS) of protons situated at the end of the pinch column. Note that the parameters $E_{\mathrm{d}}$ and $\phi_{0}$ are relevant only to the BT calculation. Histograms of radial track density were obtained, both for the experimental data and the simulation results, by sub-dividing the circular exposed area into concentric annular rings of $0.5 \mathrm{~mm}$ width. Each bin of the histogram contains the areal track density for the corresponding ring.

The per-shot average radial distribution obtained from experiment, together with best-fit simulation results (for $E_{\mathrm{d}}=50 \mathrm{keV}$ ) are shown in Fig. 4. The experimental data points represent the cumulative track density distribution for all 18 exposed detectors divided by 119 - the total number of shots. The statistical uncertainty in all but one of the experimental data point are in the range 1.5 to $2.0 \%$, the exception being the $0.5 \mathrm{~mm}$ data point with an uncertainty of $2.3 \%$. The statistical uncertainties in the Monte Carlo simulation "data points" are typically an order of magnitude smaller. 


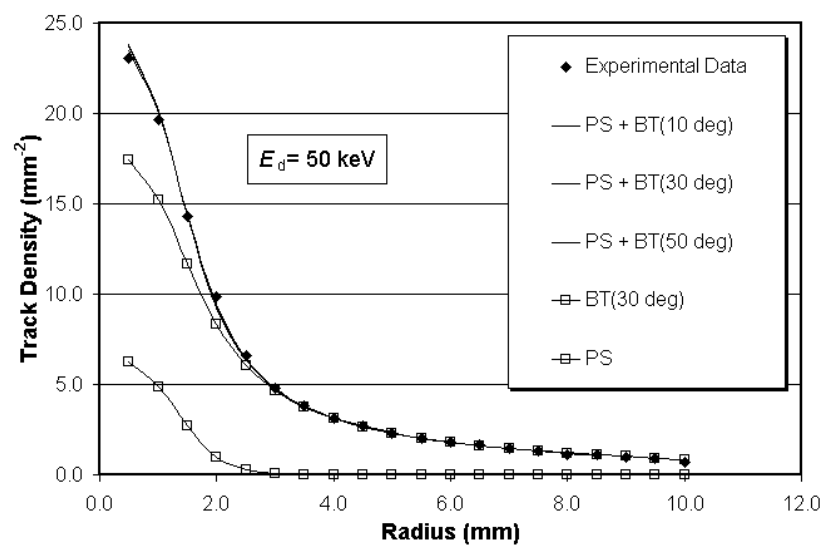

Figure 4. Experimental Data points $(\diamond)$ are per-shot average radial track density data. Lines are results of Monte Carlo simulations for deuteron source energy $E_{\mathrm{d}}=50 \mathrm{keV}$, and emission cone half-angle $\phi_{0}$ of 10,30 and 50 degrees. Error bars omitted for clarity.

Several experiments on small PF devices[3, 14, 16], have indicated that the bulk of fusion production is due to deuterons with initial energies in the $40-70 \mathrm{keV}$ range, emitted in a beam of fairly large angular width $\left(\sim 30^{\circ}\right)$, and this has guided our choice of $E_{\mathrm{d}}$ and $\phi_{0}$ for performing simulations. It is found that a good fit can be obtained between the experimental and calculated radial distributions for a fairly wide range of deuteron energies and cone angles: $E_{\mathrm{d}}=40-70 \mathrm{keV}$ and $\phi_{0}=10^{\circ}-50^{\circ}$, as simulation input- but only if a linear combination of the BT and PS distributions is used. The best-fit was obtained by minimising the chi-squared parameter. For example, Table I shows the fits obtained for $E_{\mathrm{d}}=50 \mathrm{keV}$ and a range of cone halfangles $\phi_{0}$. These fits are presented as the first three curves (without point symbols) in Fig. 4, and they lie very nearly on top of one another. The last two curves (with square point symbols) represent the separate BT and PS distributions for the case of $E_{\mathrm{d}}=50 \mathrm{keV}$ and $\phi_{0}=30^{\circ}$, for which PS fusion contributes a little less than $6 \%$ of the total neutron yield. As expected the PS distribution is practically zero beyond a radius of $2 \mathrm{~mm}$. It is especially clear from Fig. 4 and Table I, that models which suppose the fusion to occur entirely within the pinch region or on the $\mathrm{PF}$ axis (e.g. thermonuclear or moving boiler models - characterised by a PS shaped distribution) are in marked disagreement with the experimental data presented here.

Table I. Best-fits to experimental data for source deuteron energy $E_{\mathrm{d}}=50 \mathrm{keV}$, and three values of BT fusion cone half-angle $\phi_{0}$. PS \% is the percentage of the total neutron yield derived from the point-source (i.e. pinch) fusion contribution. $N_{\mathrm{d}}$ is the number of deuterons emitted isotropically within the BT cone and $Y_{\mathrm{n}}$ is the associated total neutron yield.

\begin{tabular}{cc|cc|cr|c}
\hline$E_{\mathrm{d}}$ & $\phi_{0}$ & \multicolumn{6}{c}{ Beam-Target Source } & \multicolumn{2}{c}{ Point Source } & Total \\
$(\mathrm{keV})$ & $(\mathrm{deg})$ & $N_{\mathrm{d}}\left(\times 10^{15}\right)$ & $Y_{\mathrm{n}}^{\text {BT }}\left(\times 10^{7}\right)$ & $Y_{\mathrm{n}}^{P S}\left(\times 10^{7}\right)$ & \multicolumn{1}{c}{$P S \%$} & $Y_{\mathrm{n}}\left(\times 10^{7}\right)$ \\
\hline \multirow{3}{*}{50} & 10 & 1.49 & 1.54 & 0.21 & $12.0 \%$ & 1.75 \\
& 30 & 3.99 & 4.24 & 0.26 & $5.8 \%$ & 4.50 \\
& 50 & 6.69 & 6.08 & 0.28 & $4.4 \%$ & 6.36 \\
\hline \multirow{3}{*}{70} & 10 & 0.65 & 1.51 & 0.28 & $15.6 \%$ & 1.79 \\
& 30 & 1.85 & 4.42 & 0.30 & $6.4 \%$ & 4.72 \\
& 50 & 3.06 & 6.36 & 0.31 & $4.6 \%$ & 6.67 \\
\hline
\end{tabular}

The reason that the experimental data can be fitted by the Monte Carlo simulation for a fairly wide choice of $E_{\mathrm{d}}$ and $\phi_{0}$, is that the shape of the BT track density distribution changes only slightly as these parameters are varied. However, the magnitude of this distribution depends strongly on the choice of $\phi_{0}$, which is reflected by the large variation in $N_{\mathrm{d}}$ and $Y_{\mathrm{n}}$ found in Table I. Of course, it is not physically plausible that the deuterons emitted from the PF pinch should be mono-energetic, or that the emission be perfectly isotropic within a sharply defined cone; rather the $E_{\mathrm{d}}$ and $\phi_{0}$ values input to the Monte Carlo simulation can be thought of as characteristic values. Also, the neutron yields shown in this table are smaller than the per-shot average yield as determined from the indium activation counter $\left(Y_{\mathrm{n}} \approx 10^{8}\right)$. The most likely explanation for this disparity is that the sensitivity of our activation counter has drifted since its calibration several years ago.

The percentage of point-source PS fusion required in order to obtain the best fit between simulated and experimental radial distributions is shown, as a function of both $E_{\mathrm{d}}$ and $\phi_{0}$, in Fig. 5. It is evident that the PS contribution required lies in the 4 to $6 \%$ range for quite a wide selection of $E_{\mathrm{d}}$ and $\phi_{0}$ values. The flatteningout of this surface for larger values of $\phi_{0}$ (i.e. wider cone half-angle of deuteron emission) suggests that the minimum point-source contribution required to fit the experimental data is about $4 \%$. On the other hand, a PS contribution above $10 \%$ implies a fairly narrow emission cone $\phi_{0} \leq 15^{\circ}$ and a deuteron energy of $E_{\mathrm{d}} \geq 50 \mathrm{keV}$. However, such a narrow emission cone implies an average neutron yield that is very much less than $10^{8} /$ shot (see Table I), which would signify a rather large error in the value determined from our activation detector. 
Therefore, it seems reasonable to conclude that beamtarget fusion represents the predominant ( $90 \%$ or more) contribution to the total neutron yield.

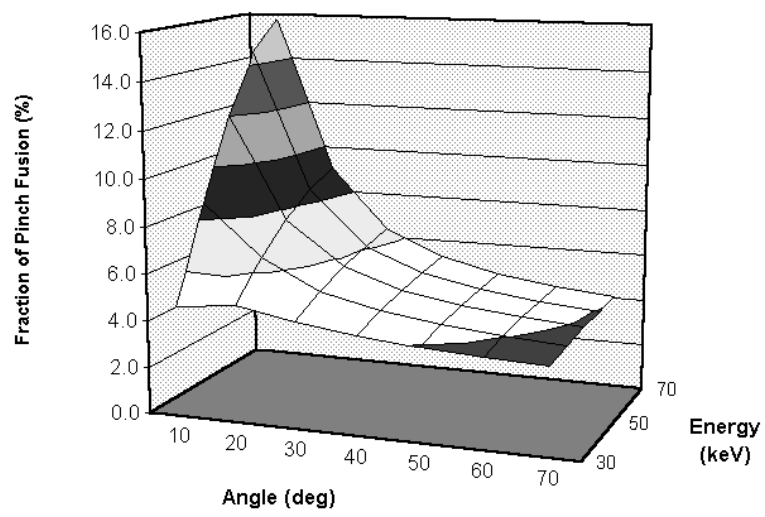

Figure 5. Surface plot of percentage of point-source fusion (PS) as a function of $E_{\mathrm{d}}$ and $\phi_{0}$, required to obtain the best-fit between simulation and experimental radial distributions. The white part of the surface represents the band of 4 to $6 \%$ point-source contribution.

As a last point, it is interesting to note that the total energy associated with the deuteron beam is quite significant and depends strongly on the value of $E_{\mathrm{d}}$. For example, for the $E_{\mathrm{d}}=50 \mathrm{keV}$ and $\phi_{0}=30^{\circ}$ case as shown in Table I, the number of deuterons in the beam is calculated to be $3.99 \times 10^{15}$ which corresponds to a total beam energy of $32 \mathrm{~J}$ (i.e. about $1 \%$ of the energy stored in the capacitor-bank), and the associated beam-target yield is $4.24 \times 10^{7}$ neutrons. For ease of comparison, beam energies can be scaled to a fixed BT yield of $10^{8}$ neutrons per shot; then the total beam energy as a function of $E_{\mathrm{d}}$, as calculated from our Monte Carlo simulation code, is shown in Fig. 6.

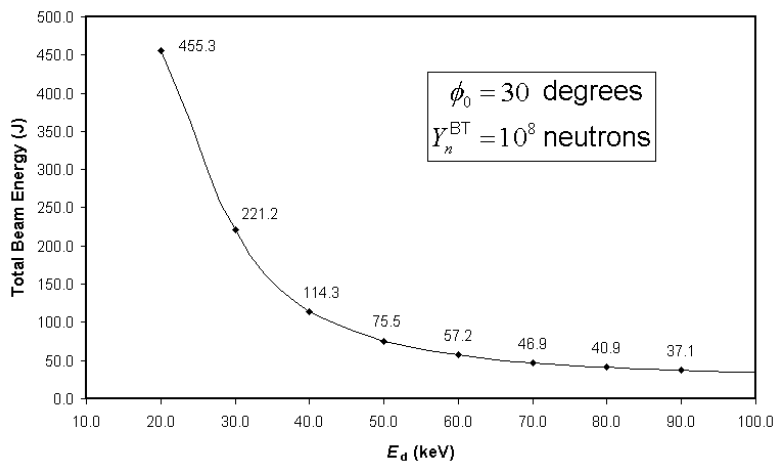

Figure 6. Graph of the simulation-calculated total deuteron beam energy associated with a yield of $10^{8}$ neutrons versus the initial deuteron emission energy $E_{\mathrm{d}}$ (for the $\phi_{0}=30^{\circ}$ case, with our plasma focus geometry). Data points at $E_{\mathrm{d}}=20,30, \ldots, 90 \mathrm{keV}$ are labelled with calculated values of total beam energy.

It is clear from this figure that-for a given BT neutron yield - the fraction of the bank energy that must be converted into deuteron beam energy increases rapidly as $E_{\mathrm{d}}$ drops below $40 \mathrm{keV}$; this is a result of the rapidly decreasing ${ }^{2} \mathrm{H}(d, n)^{3} \mathrm{He}$ reaction cross-section at lower energies. Although the conversion efficiency of stored bank energy to ion beam energy in the PF is not known with any accuracy, this calculation does lend support to the conclusion of several studies with small PF devices, that deuterons with initial energies in the $40-70 \mathrm{keV}$ range are responsible for the bulk of the neutron yield $[3,14,16]$.

\section{Conclusions}

A camera obscura technique has been used to investigate the spatial distribution of fusion within the plasma focus by imaging the proton yield from the ${ }^{2} \mathrm{H}(d, p)^{3} \mathrm{H}$ reaction. Our automated detector scanning system measured and recorded multi-parameter data for a large number of candidate features on the surface of more than twenty PM-355 detectors. By applying selection criteria to this feature data, a very good separation between spurious "tracks" and genuine fusion proton tracks was achieved. The filtered proton data from 18 exposed detectors was then combined to obtain a per-shot averaged track density profile (i.e. areal track density versus radius). This experimental profile has been compared to the results of a detailed Monte Carlo simulation which attempts to include all physically significant factors, such as: fusion reaction total and differential cross-sections, centre-of-mass to lab-frame transformation, energy-loss along deuteron trajectories, geometry of PF chamber and camera obscura, and scattering in the kapton window. The comparison between simulation and experimental data yields significant evidence that beam-target fusion is the dominant mechanism for small PF devices. In particular the width and shape of the experimentally observed track density profile is not explicable in terms of a pure thermonuclear or moving-boiler model, in which all the fusion is localised on the PF axis. However there is clear evidence that 4 to $10 \%$ of the yield comes from fusion in the vicinity of the plasma focus pinch. Essentially no proton energy information is obtained in this measurement, and so it is not possible to determine the nature of the fusion mechanism, e.g. thermonuclear, gyrating-particle, or some other mechanism.

The results indicate that the beam-target mechanism produces 90 to $96 \%$ of the total fusion yield in our device, and it is distributed over a conical volume with a fairly wide angle. A better calibrated neutron detector than the one available, will be required to determine the width of the cone more accurately. A reasonably defensible choice of initial deuteron energy and cone of half-angle would be $E_{\mathrm{d}}=50 \mathrm{keV}, \phi_{0}=30^{\circ}$ plus a $6 \%$ point-source contribution. This case gives a good fit to our experimental data; it lies within the plateau region of Fig. 5; and it is consistent with a number of 
other experimental PF fusion studies.

Solid-state nuclear track detectors (particularly CR39/PM-355), used in combination with an automated scanning system, are a powerful tool for the investigation of plasma focus fusion. Measurements of the charged fusion products - protons, tritons and ${ }^{3} \mathrm{He}-$ are not only complementary, but in many ways more informative than the more common neutron measurements.

\section{Acknowledgements}

This work was supported by the Academic Research Fund, National Institute of Education, Nanyang Technological University, Singapore, RP 6/96 VS. The first author would like to thank the participants at both, the $1^{\text {st }}$ IAEA Technical Committee Meeting on Applications of Fusion Energy to Science and Technology (30 Oct -3 Nov 2000, Chengdu, China), and the $14^{\text {th }}$ IAEA Technical Committee Meeting on Research Using Small Fusion Devices (25 - 27 June 2001, Sao Pãulo , Brazil) for their valuable suggestions and comments.

\section{References}

[1] N.V. Filipov, T.L. Fillipova, and V.N. Vinogradov, Nuclear Fusion 2, 577 (1962).

[2] J.W. Mather, Phys. Fluids 8, 528 (1964).

[3] I. Tiseanu, N. Mandache and V. Zambreanu, Plasma Phys. Control. Fusion 36, 417 (1994).
[4] M.J. Bernstein and G.G. Comisar, Phys. Fluids 15, 700 (1972).

[5] U. Jäger and H. Herold, Nucl. Fus. 27, 407 (1987).

[6] A. Bernard, A. Coudeville, A. Jolas, J. Launspach and J. de Mascureau, Phys. Fluids 18, 180 (1975).

[7] K. Steinmetz, K. Hubner, J.P. Rager and B.V. Robouch, Nuclear Fusion 22, 25 (1982).

[8] U. Jäger, L. Bertalot and H. Herold, Rev. Sci. Instrum. 56, 77 (1985).

[9] S.A. Durrani and R.K. Bull, Solid State Nuclear Track Detection (New York, Pergamon Press, 1987).

[10] A. Szydlowski, M. Sadowski, T. Czyzewski, M. Jaskla and A. Korman, Nucl. Instr. and Meth. B149, 113 (1999).

[11] S. Lee, T.Y. Tou, S.P. Moo, M.A. Eissa, A.V. Gholap, K.H. Kwek, S. Mulyodrono, A.J. Smith, Suryadi, W. Usada and M. Zakaullah, Am. J. Phys. 56, 62 (1988).

[12] S. Lee, S.P. Moo, C.S. Wong and A.C. Chew, International Centre for Theoretical Physics (Trieste), ICTP Report. No. IC/98/231 (1998).

[13] J.F. Ziegler and J.M. Manoya, Nucl. Instr. and Meth. B35, 215 (1988).

[14] S.V. Springham, S. Lee and M.S. Rafique, Plasma Phys. Control. Fusion 42, 1023 (2000).

[15] R.E. Brown and N. Jarmie, Phys. Rev. C41, 1391 (1990).

[16] H. Kelly and A. Marquez, Plasma Phys. Control. Fusion 38, 1931 (1996). 